\title{
SYNTHESIS AND BIOLOGICAL ACTIVITY OF SPIRO[BENZO[H]QUINAZOLINE-5,1'-CYCLOHEPTANE]- 4(6H)-ONE DERIVATIVES
}

\section{A.S. Ayvazyan', A.I. Markosyan', F.H. Arsenyan' ${ }^{1}$ and Kh.S. Hakobyan'}

${ }^{1}$ Scientific and Technological Center of Organic and Pharmaceutical Chemistry of NAS RA Armenia, 0014, Yerevan, Azatutyan ave. 26. Phone: +374 10288443.

DOI: 10.19163/MedChemRussia2021-2021-240

E-mail: ani.ayvazyan17@mail.ru

As a result of the interaction of ethyl 4'-amino-1'H-spiro[cycloheptane-1,2'naphthalene]-3'-carboxylate [1] and benzoyl isothiocyanate, followed by cyclization of the resulting thiourea, 2-thioxo-2,3-dihydro-1H-spiro[benzo[h]quinazoline5,1 -cycloheptan]-4(6H)-one (2). Condensation of the latter with halides of various structures are synthesized2-thiosubstituted 3H-spiro[benzo[h]quinazoline-5,1'cycloheptan]-4(6H)-ones (3), 2,2'-(methylenebis(sulfanediyl))bis(3H-spiro[benzo[h] quinazoline-5,1'-cycloheptan]-4(6H)-one) (4), 9,10-dihydrospiro[benzo[h] thiazolo[2,3-b]quinazoline-6,1'-cycloheptan]-7(5H)-ones (5) and 10,11-dihydro-5Hspi-ro[benzo[h][1,3]thiazino[2,3-b]quinazoline-6,1'-cycloheptan]-7(9H)-one (6).

Studied antitumor properties against sarcoma 180 and antibacterial properties of the synthesized compounds. Gram-positive (Staphylococcus aureus 209p, Bacillus subtilis ATCC 6633) and gram-negative microorganisms (Sh. Flexneri 6858, E. coli 0-55) were used as test objects.

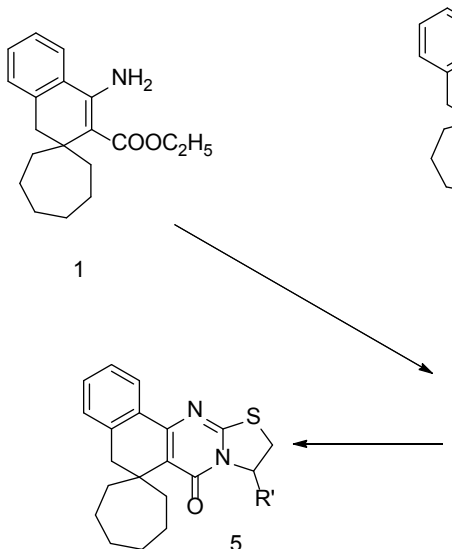

5<smiles>[R]Sc1nc2c(c(=O)[nH]1)C1(CCCCCC1)Cc1ccccc1-2</smiles><smiles>O=c1[nH]c(=S)[nH]c2c1C1(CCCCCC1)Cc1ccccc1-2</smiles><smiles>O=c1[nH]c(SCSc2nc3c(c(=O)[nH]2)C2(CCCCCC2)Cc2ccccc2-3)nc2c1Cc1ccccc1-2</smiles>
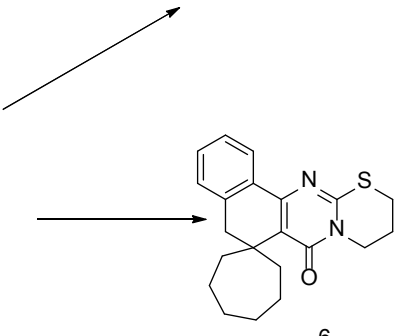

\section{References}

[1]A. I. Markosyan, A. S. Ayvazyan, S. H. Gabrielyan, S. S. Mamyan. C Chem J. Armenia. 2019, v. 72 (4), p. 469-482. 